\title{
Overview of Lung Development in the Newborn Human
}

\author{
David Warburton
}

NHLBI LungMAP Project, The Saban Research Institute, Children's Hospital Los Angeles, Keck School of Medicine and Ostrow School of Dentistry, University of Southern California, Los Angeles, CA, USA

\section{Keywords}

Lung development · Human · Newborn infant

\begin{abstract}
In human neonates rapid adaptation from an aqueous intrauterine environment to permanent air breathing is the ratelimiting step for extrauterine life, failure of which justifies the existence of neonatal intensive care units. The lung develops at about 4-6 weeks' gestation in humans as a ventral outpouching of the primitive foregut into the surrounding ventral mesenchyme, termed the laryngotracheal groove. At its posterior end lie progenitor cells that form a pair of bronchial tubes, from which arise all the distal epithelial structures of the lung. In humans, formation of the distal gas exchange surfaces begins in utero at about 20 weeks' gestation and is substantially established by term. Stereotypic branching of the proximal airway ends relatively early at 16-18 weeks at the bronchoalveolar duct junctions. Distally, about 5 finger-like alveolar ducts arise from each bronchoalveolar duct junction and ramify outwards towards the pleura. The majority of alveolar air sacs arise from the sides of the alveolar ducts and each of these alveoli can have up to 5 daughter alveoli arising from the outer surface as subsequent buds. At the end of each alveolar duct lie the mouths of 5 intercon-
\end{abstract}

\section{KARGER}

(C) 2017 S. Karger AG, Basel

E-Mail karger@karger.com

www.karger.com/neo nected alveoli. Each family of alveoli arising from each bronchoalveolar duct junction has a different shape depending upon the limitations imposed by the pleural surface as well as the interstitial fascial planes.

(c) 2017 S. Karger AG, Basel

The evolution of the lung is what separates the larger air-breathing terrestrial animals from fish. Similarly, in human neonates it is the one-way rapid adaptation from an aqueous intrauterine environment to permanent air breathing that is the rate limiting step for extrauterine life, failure of which justifies the existence of neonatology as a medical specialty as well as neonatal intensive care units (NICUs).

The lung develops at about 4-6 weeks' gestation in humans as a ventral outpouching of the primitive foregut into the surrounding ventral mesenchyme. This structure is termed the laryngotracheal groove and it is the forerunner of the larynx and trachea. At the posterior end of this structure a set of progenitor cells arise which form a pair of tubes, from which arise all the distal epithelial structures of the lung. In mice, mutations of the retinoic acid receptor transcription factor activation pathway interfere with formation of the laryngotracheal groove, whereas

Prof. David Warburton

The Saban Research Institute, Children's Hospital Los Angeles, Keck School of Medicine Ostrow School of Dentistry, University of Southern California

4650 Sunset Boulevard, Los Angeles, CA 90027 (USA)

E-Mail dwarburton@chla.usc.edu 
mutations of the transcription factor $\mathrm{Nkx} 2.1$ as well as in the fibroblast growth factor (FGF) ligand and receptor pathway prevent lung epithelial morphogenesis distal to the trachea and primary bronchi $[1,2]$.

Epithelial branching morphogenesis distal to the carina thus clearly depends on these signaling processes as well as being modulated by several other known modes of epithelial-mesenchymal crosstalk. The lung mesenchyme contains various kinds of fibroblasts as well as vascular and lymphatic endothelial and neural networks, along with a complex extracellular matrix. Interference with any or all of these cellular functions disrupts lung branching and, hence, eventually the development of the gas exchange surface (GES).

Primary and subsequent branching of the lung has been described as orthogonal, i.e., branches which arise from the sides of bronchi rather like bristles on a bottle brush, planar, i.e., divarication of the tips of branches in 1 plane, and rotational, which means division of tips in the plane at right angles to a former pair of branches. Therefore, a popular hypothesis for many years has been that mesenchymally derived morphogens such as FGFs, particularly FGF10 in mice, act as chemoattractants to induce migration of the tip cells to form branches, much as occurs in the trachea of the fruit fly Drosophila, in a conserved response to FGF signaling $[3,4]$.

However, our more recent work shows that things are actually not quite so simple as this and importantly that scaling of the lung between mice and humans differs considerably. For example, loss of function mutations in the FGF pathway in humans does not usually present as neonatal lethal pulmonary problems, but as lacrimo-auriculo-dento-digital (LADD syndrome), suggesting evolutionary divergence of the clock mechanism that regulates branching of the airway. In other words, mice have shorter interbranch lengths and thus branch quickly a small number of times, whereas interbranch lengths are much longer and the number of branches is greater in humans (one wonders how this compares with animals with much bigger chests like whales, giraffes, horses, and Diplodocus). We think this adaptation came about because the human lung is much larger than the mouse lung and, therefore, there is much more scalar space to fill with branches and GES.

In flies as well as mice, airway branching is regulated by intracellular calcium waves that propagate via intercellular connections. In the fly, there is no airway smooth muscle, so calcium waves, mediated by calcium uptake into the mitochondria via the SERCA (sarcoendoplasmic reticulum calcium-ATPase) calcium pump, set up distal

Overview of Lung Development in the

Newborn Human to proximal gradients between the distal leading tip cells in the trachea (high) and their followers (low). In the mouse, the calcium gradients are driven by pacemakers located in the hilum of the lung and process distally down the smooth muscle layer of the airway, resulting in peristaltic waves of muscle contraction. Inhibition of SERCA results in the reversible inhibition of these calcium waves and, with that, reversible inhibition of peristalsis as well as branching morphogenesis of the airway. We can also see these waves of peristalsis occurring in early human fetal lung, with the additional recent discovery that strong contractions of the interbranch smooth muscle appears to physically drive the division of the distal branch tips [5; unpubl. data].

Thus, the branch stalks as well as the branch tip interclefts are invested with smooth muscle cells that undergo rhythmic calcium pump-driven peristaltic contractions that spread distally and end as intercleft contractions. The distribution of the smooth muscle can thus be likened to a sleeve ending in a glove without fingers. Branches only appear where smooth muscle is not present.

We have put some considerable effort into considering the physics and mathematics of these peristaltic waves and how they might be integrated with biology to drive stereotypic airway branching. These waves of peristalsis move fluid within the airway lumen and, by hydraulic forces, dilate the epithelial distal tips of the airway as well as epithelium overlying lateral holes in the smooth muscle. Thus, we now hypothesize that hydraulic pressure waves driven by these contractions look to be substantially responsible for outward buckling of the epithelium at weak points where smooth muscle is not, to form new branches, which in turn acquire a smooth muscle coat until the point where the next branch will occur and so on. Moreover, there is a fine network of neurons within thelung, the function of which is also essential for branching morphogenesis [6-9].

In mice, formation of the distal GES occurs postnatally, whereas in humans formation of the GES begins in utero at about 20 weeks' gestation and is substantially established by 40 weeks at term. This is another important developmental difference between mice and humans. Yet in both species, stereotypic branching of the proximal airway ends relatively early in utero at the bronchoalveolar duct junctions (BADJs). Distal to the BADJs the alveolar ducts extend outwards towards the pleura. About 5 finger-like alveolar ducts arise from each BADJ and they appear to ramify into the mesenchyme rather like a rabbit warren. The majority of GES arises from the sides of the alveolar ducts and each of these al- 
veolar buds can have up to 4 or 5 daughter alveoli arising from the outer surface as subsequent buds. At the end of each alveolar duct lie the mouths of 5 or so interconnected alveoli. Each family of alveoli arising from each BADJ has a different shape depending upon the limitations imposed by the pleural surface as well as the interstitial fascial planes. We think that the eventual number of alveoli may be governed by the mathematical packing rules of physics, rather like Newton's kissing theorem for billiard balls in a frame.

Alveolarization is well established in humans by 40 weeks (term gestation). The major challenge for term human infants at the birth transition is the rapid evacuation of intraluminal fluid. The fetal lung secretes a salty fluid that flows up and out of the trachea and larynx as a consequence of active chloride secretion by the lung epithelium. Following separation of the placenta and/or clamping of the umbilical cord there is a massive outpouring of epinephrine and norepinephrine from the newborn's adrenal glands. The beta adrenergic effect of this adrenal surge is to substantially switch over chloride secretion from the alveolar epithelium in favor of active absorption of sodium and hence water, mediated by beta-adrenergic activation of the enzyme $\mathrm{Na} / \mathrm{K}$ ATPase. Thus, intraluminal water follows sodium and is quite rapidly absorbed into the pulmonary interstitium, so fast in fact that cuffs of interstitial fluid are found surrounding the pulmonary veins and lymphatics. A large part of this fluid then proceeds to drain quite rapidly up the pulmonary lymphatic trunks, entering the central venous circulation via the thoracic duct. Delay of the fluid absorption process or indeed malfunction or mutation of the $\mathrm{Na} / \mathrm{K}$ ATPase pathway may present as transient tachypnea of the newborn $[1,2]$.

Maturation of the surfactant system increases between about 30 weeks' gestation and term in humans under the control of a surge in fetal adrenal cortisol production. Humans born prior to about 34-36 weeks' gestation may present with respiratory distress syndrome that can be substantially corrected with surfactant treatment.

When humans are born prior to 30 weeks' gestation they have a deficiency of GES as well as of surfactant. Prior to about 28 weeks' gestation there may be no mature GES with which to breathe; instead merely alveolar ducts and a few rudimentary alveolar buds are present. Furthermore, major components of the mesenchyme-like vascular and lymphatic endothelial as well mesenchymal matrix component networks will be poorly developed. Moreover, at this early stage of gestation, delivery into room air represents a major oxidative stress.
As mentioned above, early maturation of the GES in humans occurs under a positive hydraulic pressure environment with a laryngeal occlusive positive end-expiratory pressure of about $2.5 \mathrm{~cm}$ of water, with positive hydraulic pressure peristalsis of the airway, superimposed on occasional waves of negative pressure imposed by fetal breathing movements of the diaphragm. Following delivery, any subsequent maturation of the alveolar buds occurs in an air-filled negative pressure breathing environment. Therefore, it is not surprising that following delivery of extremely preterm human infants, particularly when they require positive pressure ventilation, oxygen therapy and/or excess circulatory loading with fluid, GES maturation goes awry, leading to arrest or "simplification" of the GES. This pathobiology has been dubbed by Alan Jobe as the "new form" of bronchopulmonary dysplasia, to be distinguished from the "old form" which was primarily a sequela of surfactant deficiency, the resulting respiratory distress syndrome, as well as superimposed oxygen and ventilation therapy in infants greater than 30-32 weeks' gestation, and was marked by interstitial edema and fibrosis [10].

Now perhaps we can understand why, at present, the best we can hope for is that in extreme prematurity in the NICU, gentler ventilation strategies, tolerance for less oxygen and judicious thermoregulatory, fluid, electrolyte and nutritional management may allow the lung to reentrain its natural capacity to mature the GES into something resembling a normal configuration. That survivors of the NICU are now arriving in young adulthood suggests there is reason to be optimistic about postnatal recovery and specifically the growth recovery of the lung, with significant expansion of the lung GES functional reserve. However, the fact that survivors of BPD can desaturate on maximal exercise suggests that the capacity for postnatal recovery of lung function may be limited in many cases. Nevertheless, we do not yet know what exactly are the detailed effects of human prematurity on the development of the lung in vivo in humans, nor do we know what the scope of the potential developmental functional GES reserve may be, nor indeed how best to optimize it.

\section{Disclosure Statement}

The author has no conflicts of interest to declare. 


\section{References}

1 Warburton D, El-Hashash A, Carraro G, Tiozz Co, Sala F, Rogers O, De Langhe S, Kemp PJ, Riccardi D, Torday J, Bellusci S, Shi W, Lubkin SR, Jesudason E: Lung organogenesis. Curr Top Dev Biol 2010;90:73-158.

$\checkmark 2$ Warburton D, Bellusci S, Del Moral P-M, Kaartinen V, Lee M, Tefft D, Shi W: Growth factor signaling in lung morphogenetic centers: automaticity, stereotypy and symmetry. Respir Res 2003;4:5.

>3 Metzger RJ, Klein OD, Martin GR, Krasnow MA: The branching program of mouse lung development. Nature 2008;453:745-750.
4 Warburton D: Order in the lung. Nature 2008;453:733-735.

5 Jesudason EC, Keshet E, Warburton D: Entrained pulmonary clocks: epithelium and vasculature keeping pace. Am J Physiol Lung Cell Mol Physiol 2010;299:L453-L454.

-6 Guo Y, Chen T-H, Zeng X, Warburton D, Boström KI, Ho C-M, Zhao X, Garfinkel A: Branching patterns emerge in a mathematical model of the dynamics of lung development. J Physiol 2014;592:313-324.

7 George UZ, Bokka KK, Warburton D, Lubkin SR: Quantifying stretch and secretion in the embryonic lung: implications for morphogenesis. Mech Dev 2015;138:356-363.
$>8$ Bokka KK, Jesudason EC, Lozoya OA, Guilak F, Warburton D, Lubkin SR: Morphogenetic implications of peristalsis-driven fluid flow in the embryonic lung. PLoS One 2015;10: e0132015.

9 Bokka KK, Jesudason EC, Warburton D, Lubkin SR: Morphogenetic implications of peristaltic fluid-tissue dynamics in the embryonic lung. J Theor Biol 2015;382:378-385.

10 Jobe AH: The new bronchopulmonary dysplasia. Curr Opin Pediatr 2011;23:167-172. 